\title{
Pacific
}

Journal of

Mathematics

\section{SPECTRAL THEORY FOR LINEAR RELATIONS VIA LINEAR OPERATORS}

DANA GHEORGHE AND FlORIAN-HORIA VASILESCU 


\title{
SPECTRAL THEORY FOR LINEAR RELATIONS VIA LINEAR OPERATORS
}

\author{
DANA GHEORghe AND Florian-Horia VASILESCU
}

\begin{abstract}
We develop a spectral theory for closed linear operators of the form $T$ : $D(T) \subset X \mapsto X / X_{0}$, where $X$ is a complex Banach space and $X_{0}$ a closed vector subspace of it. This approach, essentially expressed in terms of linear operators, provides a better understanding of the spectral theory for closed linear relations.
\end{abstract}

\section{Introduction}

As in the case of linear operators, the spectral theory of linear relations, including the associated analytic functional calculus, is an important tool for studying various properties of these objects and for deriving some of their applications. Results related to the spectral theory of linear relations and its applications can be found in [Baskakov and Chernyshov 2002; Baskakov and Zagorskiî 2007; Cross 1998; Favini and Yagi 1993; Favini and Yagi 1999] and elsewhere.

In this paper we emphasize the strong connection between the spectral theory of closed linear relations and that of some closed linear operators. As a matter of fact, we develop a spectral theory for a certain class of linear operators, obtaining as consequences most of the main spectral properties of linear relations.

Our concept of spectrum is equivalent to that of extended spectrum of a linear relation, as given by [Baskakov and Chernyshov 2002, Definition 1.5]; see also [Cross 1998, Section VI.4], where it is called augmented spectrum. In particular, the point $\infty$ is in the spectrum unless the quotient range operator is an ordinary everywhere-defined bounded operator (see Proposition 11).

Let us introduce some notation and definitions.

Let $X$ be a complex Banach space and let $\mathscr{B}(X)$ denote the Banach algebra of all bounded linear operators from $X$ into $X$. Let also $X_{0} \subset X$ be a closed vector subspace, and let $J_{0}: X \mapsto X / X_{0}$ be the canonical projection. The identity operator on $X$ will be usually denoted by $I$.

D. Gheorghe gratefully acknowledges support from the grant PN-II-RU-PD-2011-3-0052 (CNCSRomania).

MSC2010: primary 47A06, 47A10, 47A60; secondary 47A56.

Keywords: linear relations, spectrum, analytic functional calculus. 
We are interested in linear operators of the form $T: D(T) \subset X \mapsto X / X_{0}$, where $D(T)$ is, of course, the domain of $T$. (The use of such operators is inspired by the works [Albrecht and Vasilescu 1986; Waelbroeck 1982]; see also [Gheorghe and Vasilescu 2009].) Such an operator is said to be a quotient range operator. Although $X / X_{0}$ is itself a Banach space, its quotient space form plays an important role in what follows. As a matter of fact, the class of closed quotient range operators is in one-to-one correspondence with the class of closed linear relations (see the definition below), and they have important similar properties. Note that the formula $T: D(T) \subset X \mapsto X / X_{0}$ implies that $T$ is a quotient range operator, and in such situations the expression "quotient range" will be often omitted.

If $T: D(T) \subset X \mapsto X / X_{0}$, we denote, as usual, by $N(T), R(T)$ and $G(T)$ the null-space, the range and the graph of $T$. Let $R_{0}(T)$ be given by $R(T)=R_{0}(T) / X_{0}$, and $G_{0}(T)=\left\{(x, y) \in X \times X ; x \in D(T), J_{0}(y)=T(x)\right\}$, which are called, with the terminology of [Albrecht and Vasilescu 1986], the lifted range and lifted graph, respectively.

Following Arens [1961], any linear subspace $Z$ of $X \times X$ is called a linear relation in $X$. Given a linear relation $Z \subset X \times X$, we associate it, as usual (see [Arens 1961; Cross 1998]), with the following subspaces:

$$
\begin{array}{ll}
D(Z)=\{u \in X ;(u, v) \in Z \text { for some } v \in X\}, & N(Z)=\{u \in D(Z) ;(u, 0) \in Z\}, \\
R(Z)=\{v \in X ;(u, v) \in Z \text { for some } u \in X\}, & M(Z)=\{v \in R(Z) ;(0, v) \in Z\} .
\end{array}
$$

The left two are called the domain of $Z$, the range of $Z$; the right are the kernel of $Z$ and the multivalued part of $Z$. When $M(Z)=\{0\}$, then $Z$ is the graph of a linear operator. We often identify the relation given by the graph of an operator with the operator itself.

Given an arbitrary relation $Z \subset X \times X$, to avoid any confusion with the inverse of an operator, we will denote the reverse relation $\{(y, x) \in X \times X ;(x, y) \in Z\}$ by $Z^{\dagger}$.

The strong connection between linear relations and quotient range operators is well known and easily explained; see [Cross 1998; Gheorghe and Vasilescu 2009] for example. Namely, given an operator $T: D(T) \subset X \mapsto X / X_{0}$, the space $Z_{T}=$ $G_{0}(T) \subset X \times X$ is a linear relation. Conversely, given a linear relation $Z \subset X \times X$, with $M(T)$ closed (which is automatic in the framework which will be used in the sequel), the linear operator $Q_{Z}: D(Z) \mapsto X / M(Z)$, given by $Q_{Z}(x)=y+M(Z)$ whenever $(x, y) \in Z$, is a quotient range operator. Moreover, this correspondence is one-to-one. This connection will be exploited to develop a spectral theory for linear relations. The simple but crucial remark leading to this development is that for a closed relation $Z \subset X \times X$, the reverse relation $Z^{\dagger}$ is (the graph of) a bounded operator if and only if the operator $Q_{Z}: D(Z) \mapsto X / M(Z)$ has a bounded inverse. 
Given a linear relation $Z$ and a complex number $\lambda \in \mathbb{C}$, we consider the linear relations $\lambda I-Z=\{(u, \lambda u-v) ;(u, v) \in Z\}$ and $(\lambda I-Z)^{\dagger}=\{(\lambda u-v, u) ;(u, v) \in Z\}$ (see Section 5). If we assume that $Z$ is closed, $N(\lambda I-Z)=\{0\}$ and $R(\lambda I-Z)=X$, then we have that $(\lambda I-Z)^{\dagger}$ is (the graph of) a closed everywhere-defined linear operator (which is, in general, neither surjective nor injective; see Example 32), and hence $(\lambda I-Z)^{\dagger} \in \mathscr{B}(X)$. Because the bounded operator $(\lambda I-Z)^{\dagger}$ exists if and only if the operator $\lambda J_{Z}-Q_{Z}: D(Z) \subset X \mapsto X / M(Z)$ has a bounded inverse (see Remark 4(ii)), where $J_{Z}: X \mapsto X / M(Z)$ is the canonical projection, the spectral theory of these objects can be simultaneously developed. However, in our opinion, the spectral theory of quotient range operators is easier to handle.

Our main tool is an analytic functional calculus for quotient range operators, defined in Section 2 by using the classical Riesz-Dunford-Waelbroeck integral formula; see [Dunford and Schwartz 1958; Waelbroeck 1954]. A similar formula, valid for linear relations, is also used in [Baskakov and Chernyshov 2002]. Nevertheless, an analytic functional calculus in its full generality seems to appear only in the present work.

The analytic functional calculus allows us to recapture, in terms of operators, most of the main spectral properties known for linear relations; see especially [Cross 1998; Baskakov and Chernyshov 2002]. Among some simplifications, we mention that our approach avoids the use of the concept of pseudoresolvent, as well as that of invariant subspace, as done in [Baskakov and Chernyshov 2002]. Other differences between our approach and that of the quoted works will be discussed in due course. We should also mention that a calculus with the exponential function and with fractional powers has been already used in [Favini and Yagi 1993] to obtain a Hille-Yoshida-Phillips-type theorem for linear relations.

The paper is organized as follows. In Section 2, we introduce a notion of spectrum for quotient range operators (equivalent to that for linear relations) in the Riemann sphere $\mathbb{C}_{\infty}$, and construct a functional calculus with analytic functions in neighborhoods of this spectrum. As mentioned above, our Theorem 16, asserting in particular the multiplicativity of the analytic functional calculus, seems to be new in this context (as well as in that of linear relations). In Section 3, we study quotient range operators with unbounded spectrum and nonempty resolvent set. The existence of a spectral decomposition corresponding to separate parts of the spectrum as well as a spectral mapping theorem are presented herein. In Section 4, we study the class of quotient range operators for which the point $\infty$ is an isolated point of the spectrum. In Section 5, we investigate some connections between the analytic functional calculus and the Arens polynomial calculus [Arens 1961]. 


\section{Spectrum and analytic functional calculus for closed quotient range operators}

As in the introduction, $X$ denotes a complex Banach space, $X_{0}$ a closed vector subspace of it, and $J_{0}: X \mapsto X / X_{0}$ the canonical projection. The symbol $\mathbb{C}_{\infty}$ denotes the one-point compactification of $\mathbb{C}$. We designate by $\mathscr{B}(X, Y)$ the Banach space of all bounded linear operators from $X$ into another Banach space $Y$. As usually, $\mathscr{B}(X, X)$ is denoted by $\mathscr{B}(X)$.

Let $T: D(T) \subset X \mapsto X / X_{0}$ be a closed linear operator. We denote by $\rho_{A}(T)$ the Arens resolvent set of $T$, that is, the set of those $\lambda \in \mathbb{C}$ such that $\left(\lambda J_{0}-T\right)^{-1} \in$ $\mathscr{B}\left(X / X_{0}, X\right)$. The Arens spectrum of $T$ is the set $\sigma_{A}(T):=\mathbb{C} \backslash \rho_{A}(T)$. Because $\lambda J_{0}-T: D(T) \subset X \mapsto X / X_{0}$ is closed, we have $\lambda \in \rho_{A}(T)$ if and only if $\lambda J_{0}-T$ is bijective.

Remark 1. Given two complex Banach spaces $X_{1}, X_{2}$, we denote by $X_{1} \oplus X_{2}$ their direct sum, endowed with a convenient norm, compatible with the norms of $X_{1}, X_{2}$.

Let $T_{j}: D\left(T_{j}\right) \subset X_{j} \mapsto X_{j} / X_{0 j}$ for $j=1,2$ be quotient range operators. Then the map

$$
T_{1} \oplus T_{2}: D\left(T_{1}\right) \oplus D\left(T_{2}\right) \subset X_{1} \oplus X_{2} \mapsto\left(X_{1} / X_{01}\right) \oplus\left(X_{2} / X_{02}\right)
$$

may be regarded as a quotient range operator, provided we identify the Banach space $\left(X_{1} / X_{01}\right) \oplus\left(X_{2} / X_{02}\right)$ with the Banach space $\left(X_{1} \oplus X_{2}\right) /\left(X_{01} \oplus X_{02}\right)$, using the natural isomorphism

$$
V:\left(X_{1} / X_{01}\right) \oplus\left(X_{2} / X_{02}\right) \mapsto\left(X_{1} \oplus X_{2}\right) /\left(X_{01} \oplus X_{02}\right)
$$

given by the assignment

$$
\begin{gathered}
\left(X_{1} / X_{01}\right) \oplus\left(X_{2} / X_{02}\right) \ni\left(x_{1}+X_{01}\right) \oplus\left(x_{2}+X_{02}\right) \mapsto \\
x_{1} \oplus x_{2}+X_{01} \oplus X_{02} \in\left(X_{1} \oplus X_{2}\right) /\left(X_{01} \oplus X_{02}\right) .
\end{gathered}
$$

We write

$$
T_{1} \oplus_{q} T_{2}:=V\left(T_{1} \oplus T_{2}\right) .
$$

In particular, given $T: D(T) \subset X \mapsto X / X_{0}$ closed such that there are closed vector subspaces $X_{1}, X_{2}$ of $X$ and $X_{01}, X_{02}$ of $X_{0}$ with $X=X_{1} \oplus X_{2}, X_{0}=X_{01} \oplus$ $X_{02}, D(T)=\left(D(T) \cap X_{1}\right) \oplus\left(D(T) \cap X_{2}\right)$, and closed operators $T_{j}: D\left(T_{j}\right) \subset X_{j} \mapsto$ $X_{j} / X_{0 j}$ with $D\left(T_{j}\right)=D(T) \cap X_{j}$ for $j=1,2$ and $T\left(x_{1} \oplus x_{2}\right)=V\left(T_{1} x_{1} \oplus T_{2} x_{2}\right)$ for all $x_{1} \oplus x_{2} \in D\left(T_{1}\right) \oplus D\left(T_{2}\right)$, we have $T=T_{1} \oplus_{q} T_{2}$.

Definition 2. Let $T: D(T) \subset X \mapsto X / X_{0}$ be closed. 
(1) Assume $\sigma_{A}(T)$ bounded, and let $m \geq 0$ be an integer. The point $\infty$ is said to be $m$-regular for $T$ if the set $\left\{\lambda^{1-m}\left(\lambda J_{0}-T\right)^{-1} J_{0} ;|\lambda| \geq r\right\}$ is bounded in $\mathscr{B}(X)$ for some $r>\sup _{\lambda \in \sigma_{A}(T)}|\lambda|$.

(2) If $\infty$ is not 0 -regular we put $\sigma(T)=\sigma_{A}(T) \cup\{\infty\}$.

(3) Assume $\infty$ to be 0-regular and $X_{0} \neq\{0\}$. If $T=T_{0} \oplus_{q} T_{1}, T_{0}:\{0\} \subset X_{0} \mapsto$ $X_{0} / X_{0}=\{0\}$, we put $\sigma(T)=\sigma_{A}(T) \cup\{\infty\}$; otherwise, $\sigma(T)=\sigma_{A}(T)$.

(4) If $\infty$ is 0 -regular and $X_{0}=\{0\}$, we put $\sigma(T)=\sigma_{A}(T)$.

The set $\sigma(T)$ is called the spectrum of $T$, and the set $\rho(T)=\mathbb{C}_{\infty} \backslash \sigma(T)$ is called the resolvent set of $T$.

The set $\sigma(T)$ is nonempty except for $X_{0}=X=\{0\}$ (see Proposition 7), but it may be equal to $\mathbb{C}_{\infty}$. For practical reasons, in this paper we work only with (quotient range) operators with nonempty resolvent set.

Example 3. The well-known fact that any continuous linear operator on a Banach space $X$ has a bounded spectrum is no longer true in the case of quotient range operators, as we can see in the following example.

Let $X$ be the Hilbert space of all square-summable complex sequences, let $A \in \mathscr{B}(X)$ be the shift

$$
A\left(\left(x_{1}, x_{2}, x_{3}, \ldots\right)=\left(0, x_{1}, x_{2}, \ldots\right)\right.
$$

and let

$$
X_{0}=\left\{\left(x_{1}, x_{2}, 0,0, \ldots\right): x_{1}, x_{2} \in \mathbb{C}\right\} .
$$

Consider the operator $T$ defined by $T x=A x+X_{0}$ for $x \in X$. Clearly $T$ is continuous and thus closed. We will show that $\sigma(T)$ is unbounded. Let $\lambda \in \mathbb{C}$. We have

$$
\begin{aligned}
& x \in N\left(\lambda J_{0}-T\right) \Longleftrightarrow \lambda x-A x+y=0 \quad \text { for some } y \in X_{0}, \\
& \left.\Longleftrightarrow \begin{array}{rl}
-\lambda x_{1} & =y_{1}, \\
x_{1}-\lambda x_{2} & =y_{2}, \\
x_{k}-\lambda x_{k+1} & =0 \quad \text { for } k \geq 2
\end{array}\right\} \text { for some } y_{1}, y_{2} \in \mathbb{C} .
\end{aligned}
$$

For $|\lambda|>1$ and $x_{1}, x_{2} \in \mathbb{C}$ consider $x_{3}=-x_{1} / \lambda^{2}-x_{2} / \lambda$. Then

$$
\left(-x_{1} / \lambda, x_{3}, x_{3} / \lambda, \ldots, x_{3} / \lambda^{k}, \ldots\right) \in N\left(\lambda J_{0}-T\right),
$$

which implies that $N\left(\lambda J_{0}-T\right) \neq\{0\}$ for $|\lambda|>1$. Consequently,

$$
\{\lambda \in \mathbb{C}:|\lambda|>1\} \subset \sigma(T),
$$

so $\sigma(T)$ is unbounded. 
Remark 4. (i) For a closed operator $T: D(T) \subset X \mapsto X$-in particular for an everywhere defined bounded operator on $X$ - Definition 2 provides the usual definition of the spectrum. Note that the density of $D(T)$ in $X$ is not required. For instance, if $A$ is the operator from Example 3, which is injective, and $T=A^{-1}$, then $T$ is not densely defined but $0 \notin \sigma(T)$, so $\rho(T) \neq \varnothing$.

Note also that if $0:\{0\} \subset X \mapsto X$, we have $\sigma(0)=\mathbb{C}_{\infty}$ if $X \neq\{0\}$ and $\sigma(0)=\varnothing$ if $X=\{0\}$, by Definition 2 .

If $X_{0}=X \neq\{0\}$ and $0:\{0\} \subset X \mapsto X / X_{0}=\{0\}$, then 0 is a quotient range operator, whose Arens spectrum is empty, and $\sigma(T)=\{\infty\}$, by Definition 2 .

(ii) Let $Z \subset X \times X$ be a closed relation. It is clear that the subspace $M(Z) \subset X$ is closed. As in the introduction, we consider the (quotient range) operator $Q_{Z}$ : $D(Z) \mapsto X / M(Z)$ given by $Q_{Z}(x)=y+M(Z)$ whenever $(x, y) \in Z$, which is closed.

Let $J_{Z}: X \mapsto X / M(Z)$ be the canonical projection. Given $\lambda \in \mathbb{C}$, the operator $\lambda J_{Z}-Q_{Z}$ is again closed. If $(\lambda I-Z)^{\dagger} \in \mathscr{B}(X)$, then $\lambda J_{Z}-Q_{Z}$ has an everywheredefined, and hence bounded, inverse. Indeed, $(\lambda I-Z)^{\dagger}$ exists if and only if for every $u \in X$ we can find a unique $x \in X$ such that $(x, y) \in Z$ and $\lambda x-y=u$ for some $y \in X$. Moreover, $x=0$ if and only if $u \in M(Z)$. Hence $\lambda J_{Z} x-Q_{Z} x=J_{Z} u$, showing that $\lambda J_{Z}-Q_{Z}$ is bijective.

Conversely, if $Q_{Z}$ is closed, then $Z$ is closed. In addition, if $\lambda J_{Z}-Q_{Z}$ is bijective, for every $u \in X$ we put $x=\left(\lambda J_{Z}-Q_{Z}\right)^{-1} J_{Z} u$. Then we have $\lambda x-y=u$ for some $y \in X$ with $(x, y) \in Z$, and so $(\lambda I-Z)^{\dagger}$ does exist. Evidently, $(\lambda I-Z)^{\dagger}=$ $\left(\lambda J_{Z}-Q_{Z}\right)^{-1} J_{Z}$.

From this discussion it clearly follows that we may define the Arens resolvent set and Arens spectrum of a closed relation $Z \subset X \times X$ by the equalities $\rho_{A}(Z)=$ $\rho_{A}\left(Q_{Z}\right)$ and $\sigma_{A}(Z)=\sigma_{A}\left(Q_{Z}\right)$, respectively. Similarly, we may define the resolvent set and spectrum of a closed relation $Z \subset X \times X$ via the equalities $\rho(Z)=\rho\left(Q_{Z}\right)$ and $\sigma(Z)=\sigma\left(Q_{Z}\right)$. Consequently, most of the spectral properties obtained for a quotient range operator can be translated into properties for linear relations. This definition of the spectrum of a linear relation coincides with the corresponding definition [Cross 1998, Definition VI.4.1] or [Baskakov and Chernyshov 2002, Definition 1.5], because the condition $\lim _{|\lambda| \rightarrow \infty}(\lambda I-Z)^{\dagger}=0$ is equivalent to the fact that $\infty$ is a 0 -regular point for $Q_{Z}$.

In fact, given an integer $m \geq 0$, we may say that the point $\infty$ is $m$-regular for the closed linear relation $Z$ if $\infty$ is $m$-regular for the operator $Q_{Z}$.

As an example, if $Z=\{0\} \times X(X \neq\{0\})$, then $D(Z)=\{0\}, M(Z)=X$ and $Q_{Z}:\{0\} \subset X \mapsto X / X=\{0\}$. Therefore, $\sigma(Z)=\sigma\left(Q_{Z}\right)=\{\infty\}$, as in (i).

Definition 5. Let $T: D(T) \subset X \mapsto X / X_{0}$ be closed, with $\rho_{A}(T) \neq \varnothing$. The function

$$
\rho_{A}(T) \ni \lambda \mapsto\left(\lambda J_{0}-T\right)^{-1} J_{0} \in \mathscr{B}(X)
$$


is called the resolvent (function) of $T$. We also put $R(\lambda, T)=\left(\lambda J_{0}-T\right)^{-1} J_{0}$.

As in the case of linear relations (see [Cross 1998; Favini and Yagi 1993]), we have a resolvent equation, which is very useful for the construction of the analytic functional calculus.

Lemma 6. If $\lambda, \mu \in \rho_{A}(T)$, then

$$
R(\mu, T)-R(\lambda-T)=(\lambda-\mu) R(\mu, T) R(\lambda, T) .
$$

Proof. Indeed, for all $\lambda, \mu \in \rho_{A}(T)$, we have the identity

$$
\left(\mu J_{0}-T\right)^{-1} J_{0}-\left(\lambda J_{0}-T\right)^{-1} J_{0}=(\lambda-\mu)\left(\mu J_{0}-T\right)^{-1} J_{0}\left(\lambda J_{0}-T\right)^{-1} J_{0},
$$

which is easily checked.

As in the case of ordinary operators, the resolvent set is open and the resolvent function is holomorphic on it.

Proposition 7. The resolvent sets $\rho_{A}(T)$ and $\rho(T)$ are open subsets of $\mathbb{C}$ and $\mathbb{C}_{\infty}$ respectively, and the resolvent function $\lambda \mapsto R(\lambda, T)$ is holomorphic on $\rho_{A}(T)$, with values in $\mathscr{B}(X)$, having an analytic extension to $\rho(T)$, whenever $\infty \in \rho(T)$. In particular, the spectrum $\sigma(T)$ is a closed subset of $\mathbb{C}_{\infty}$, which is nonempty provided $X_{0} \neq X$ or $X=X_{0} \neq\{0\}$.

Proof. We may assume $\rho(T) \neq \varnothing$. The proof is similar to the corresponding one for linear relations; see for instance [Cross 1998, Section VI.1]. Because of some differences, we shall sketch an appropriate proof.

Let $\lambda_{0} \in \rho(T)$. We show that there exists a neighborhood $V \subset \mathbb{C}_{\infty}$ of $\lambda_{0}$ such that $V \subset \rho(T)$. We have the following situations.

First, if $\lambda_{0}=\infty$, it follows from Definition 2 that there exists $r>0$ such that $\{|\lambda|>r\} \subset \rho(T)$.

Second, assume $\lambda_{0} \in \rho_{A}(T)$ and that $R\left(\lambda_{0}, T\right) \neq 0$. Then, if $\left|\lambda-\lambda_{0}\right|<$ $\left\|R\left(\lambda_{0}, T\right)\right\|^{-1}$, then $\lambda \in \rho(T)$ and

$$
R(\lambda, T)=R\left(\lambda_{0}, T\right)\left(I+\left(\lambda-\lambda_{0}\right) R\left(\lambda_{0}, T\right)\right)^{-1},
$$

implying, in particular, the holomorphy of $R(\lambda, T)$ in this open disc.

Third, next assume $R\left(\lambda_{0}, T\right)=0$. Then $J_{0}=0$, and so $X=X_{0}$. Moreover, $R(\lambda, T)=0$ for all $\lambda \in \mathbb{C}$.

If $X=X_{0}=\{0\}$, then $\rho_{A}(T)=\mathbb{C}, \rho(T)=\mathbb{C}_{\infty}$ by Definition 2 .

If $X=X_{0} \neq\{0\}$, then $D(T)=\{0\}$ (otherwise $\rho(T)=\varnothing$ ) and $\rho_{A}(T)=\rho(T)=\mathbb{C}$, again by Definition 2 .

Note that the assumption $X=X_{0} \neq\{0\}$ implies $\sigma(T) \ni\{\infty\}$. Finally, suppose that $X_{0} \neq X$ and $\sigma(T)=\varnothing$. Then $R(\lambda, T)$ is analytic in $\mathbb{C}$ and has an analytic 
extension at $\infty$. By Liouville's theorem, it follows that $R(\lambda, T)$ is a constant operator, say $C_{0}$. Since $\infty$ is a 0 -regular point of $T$, we must have $C_{0}=0$. Therefore, as above, $X=X_{0}$, which is not possible.

Remark 8. If $\sigma_{A}(T)$ is bounded, according to Proposition 7 we have a development in $\mathscr{B}(X)$ of the form

$$
R(\lambda, T)=\sum_{k=-\infty}^{\infty} \lambda^{k} C_{k}
$$

where the series is uniformly convergent when $r_{1} \leq|\lambda| \leq r_{2}$ for fixed $r_{2} \geq r_{1}>$ $\sup _{\lambda \in \sigma_{A}(T)}|\lambda|$. This representation shows that $\infty$ is $m$-regular for some integer $m \geq 0$ if and only if $C_{k}=0$ for all $k \geq m$. In particular, if $m \geq 2$, the point $\infty$ is $m$-regular for $T$ if and only if $\infty$ is a pole of $R(\lambda, T)$ of order $\leq m-1$. As already noted, $\infty$ is a 0 -regular point of $T$ if and only if $\lim _{\lambda \rightarrow \infty} R(\lambda, T)=0$, while $\infty$ is a 1-regular point if and only if $\lim _{\lambda \rightarrow \infty} R(\lambda, T)$ exists in $\mathscr{B}(X)$.

Henceforth, to avoid quotient range operators with empty spectrum, we assume that either $X_{0} \neq X$ or $X=X_{0} \neq\{0\}$, if not otherwise specified.

Definition 9. Let $T: D(T) \subset X \mapsto X / X_{0}$ be closed, with $\varnothing \neq \rho(T)$.

(i) We denote by $\mathcal{O}(T)$ the set of all complex-valued functions $f$, each of them defined and analytic in an open set containing $\sigma(T)$ and depending on $f$. By identifying any two functions equal in a neighborhood of $\sigma(T)$ (that is, considering $O(T)$ as the set of germs of analytic functions in neighborhoods of $\sigma(T)$ ), we may and will regard $\mathcal{O}(T)$ as an algebra.

(ii) Let $F \subset \mathbb{C}_{\infty}$ be closed and let $U$ be an open neighborhood of $F$. An admissible contour surrounding $F$ in $U$ is a finite system of rectifiable Jordan curves $\Gamma$, positively oriented, which is the boundary of an open set $\Delta \subset \bar{\Delta} \subset U$, with $\Delta \supset F$. Note that $\Gamma \cap F=\varnothing$ and that $\Gamma$ is a compact set in $\mathbb{C}$.

(iii) We define the analytic functional calculus for the quotient range operator $T$ as follows. Let $f \in \mathcal{O}(T)$. We set

$$
f(T):= \begin{cases}(2 \pi i)^{-1} \int_{\Gamma} f(\lambda) R(\lambda, T) d \lambda & \text { if } \infty \notin \sigma(T), \\ f(\infty) I+(2 \pi i)^{-1} \int_{\Gamma} f(\lambda) R(\lambda, T) d \lambda & \text { if } \infty \in \sigma(T),\end{cases}
$$

where $\Gamma$ is an admissible contour surrounding $\sigma(T)$ in the domain of definition of $f$.

Remark. Via Proposition 7, $f(T)$ is a continuous linear operator on $X$ that does not depend on $\Gamma$.

The next result seems to be new even in the context of linear relations. 
Proposition 10. For every quotient range closed operator $T$ with $\varnothing \neq \rho(T)$, the map $f \mapsto f(T)$ of $\mathscr{O}(T)$ into $\mathscr{B}(X)$ is an algebra morphism. If $\sigma(T) \ni \infty$, this morphism is unital.

Proof. Clearly the map $f \mapsto f(T)$ is linear. To prove the multiplicativity of the application $f \mapsto f(T)$, we follow the lines of [Vasilescu 1982, Proposition III.3.4], via Lemma 6.

Consider first the case $\infty \in \sigma(T)$.

Let $f, g \in \mathbb{O}(T)$ and let $U \subset \mathbb{C}_{\infty}$ be open in the domain of definition of both $f$, $g$, with $\sigma(T) \subset U$. Let $\Delta$ and $\Delta_{1}$ be open sets such that their boundaries $\Gamma$ and $\Gamma_{1}$, respectively, are admissible contours surrounding $\sigma(T)$ in $U$, and such that $\sigma(T) \subset \Delta \subset \bar{\Delta} \subset \Delta_{1} \subset \bar{\Delta}_{1} \subset U$. Then we have

$$
\begin{aligned}
& f(T) g(T) \\
& \begin{aligned}
=f(\infty) g(\infty) I+f(\infty) \frac{1}{2 \pi i} \int_{\Gamma_{1}} g(\mu) R(\mu, T) d \mu+g(\infty) \frac{1}{2 \pi i} \int_{\Gamma} f(\lambda) R(\lambda, T) d \lambda \\
\quad+\frac{1}{2 \pi i} \int_{\Gamma} f(\lambda) R(\lambda, T) d \lambda \frac{1}{2 \pi i} \int_{\Gamma_{1}} g(\mu) R(\mu, T) d \mu \\
=f(\infty) g(\infty) I+f(\infty) \frac{1}{2 \pi i} \int_{\Gamma_{1}} g(\mu) R(\mu, T) d \mu+g(\infty) \frac{1}{2 \pi i} \int_{\Gamma} f(\lambda) R(\lambda, T) d \lambda \\
+\frac{1}{2 \pi i} \int_{\Gamma} f(\lambda)\left(\frac{1}{2 \pi i} \int_{\Gamma_{1}}(\mu-\lambda)^{-1} g(\mu)(R(\lambda, T)-R(\mu, T)) d \mu\right) \\
=f(\infty) g(\infty) I+\frac{1}{2 \pi i} \int_{\Gamma} f(\lambda) g(\lambda) R(\lambda, T) d \lambda=(f g)(T),
\end{aligned}
\end{aligned}
$$

via Lemma 6 and the Cauchy formula at infinity for analytic functions.

If $\infty \notin \sigma(T)$, the proof is similar and will be omitted.

If $\sigma(T) \ni \infty$, by letting $p_{0}$ be the constant polynomial equal to 1 , we may take as $\Gamma$ the boundary of a closed disc in $\rho(T)$ (negatively oriented). Since $R(\lambda, T)$ is analytic in $\rho(T)$, it follows that $\int_{\Gamma} R(\lambda, T) d \lambda=0$, so $p_{0}(T)=I$.

The next result corresponds to [Baskakov and Chernyshov 2002, Lemma 2.2], whose proof uses an ergodic theorem from [Hille and Phillips 1957]. We give a direct proof based on Proposition 10.

Proposition 11. Given a closed operator $T: D(T) \subset X \mapsto X / X_{0}$, the spectrum $\sigma(T)$ is a bounded subset of $\mathbb{C}$ if and only if $X_{0}=0$ and $T \in \mathscr{B}(X)$.

Proof. We use some ideas from [Vasilescu 1982, Lemma III.3.5]; see also [Hille and Phillips 1957].

Assume $\sigma(T)$ bounded, and fix an $r>0$ such that $\sigma(T) \subset\{\lambda \in \mathbb{C} ;|\lambda|<r\}$. From the analyticity of the resolvent function (Proposition 7), it follows that there 
exists a sequence $\left(C_{n}\right)_{n \geq 0} \subset \mathscr{B}(X)$ such that

$$
R(\lambda, T)=\sum_{n=0}^{\infty} \lambda^{-n} C_{n} \quad \text { uniformly with respect to }|\lambda| \geq r .
$$

The operator $C_{0}$, given by the equality $C_{0}=\lim _{\lambda \rightarrow \infty} R(\lambda, T)$, is necessarily 0 because $\infty$ is 0 -regular

We define the bounded linear operators

$$
E=\frac{1}{2 \pi i} \int_{|\lambda|=r} R(\lambda, T) d \lambda \quad \text { and } \quad A=\frac{1}{2 \pi i} \int_{|\lambda|=r} \lambda R(\lambda, T) d \lambda .
$$

Because

$$
\frac{1}{2 \pi i} \int_{|\lambda|=r} \lambda^{n} d \lambda= \begin{cases}0 & \text { if } n \neq-1 \\ 1 & \text { if } n=-1\end{cases}
$$

we have

$$
\begin{aligned}
\frac{1}{2 \pi i} \int_{|\lambda|=r} \lambda^{k} R(\lambda, T) d \lambda & =\frac{1}{2 \pi i} \int_{|\lambda|=r} \lambda^{k} \sum_{n=0}^{\infty} \lambda^{-n} C_{n} d \lambda \\
& =\sum_{n=0}^{\infty}\left(\frac{1}{2 \pi i} \int_{|\lambda|=r} \lambda^{k-n} d \lambda\right) C_{n} r=C_{k+1}
\end{aligned}
$$

for all integers $k \geq 0$. Consequently $C_{1}=E$ and $A^{n}=C_{n+1}$. The same proposition shows that $E^{2}=E$ and $A^{n} E=E A^{n}=A^{n}$. On the other hand, if $|\lambda| \geq r$, then

$$
\lambda^{-1} I+\lambda^{-2} A+\cdots=(\lambda I-A)^{-1},
$$

which implies that

$$
R(\lambda, T)=E(\lambda I-A)^{-1}
$$

Let

$$
X_{1}=(I-E)(X) \quad \text { and } \quad X_{2}=E(X) .
$$

Hence $X=X_{1} \oplus X_{2}$ because $E$ is a projection. Setting $A_{2}=\left.A\right|_{X_{2}}$ and using the fact that $A E=E A$, we have

$$
\left.(\lambda I-A)^{-1}\right|_{X_{2}}=\left(\lambda I_{2}-A_{2}\right)^{-1},
$$

whenever $|\lambda| \geq r$, where $I_{2}$ is the identity on $X_{2}$. This together with (2) implies

$$
\begin{array}{ll}
R(\lambda, T)\left(X_{1}\right) \subset X_{1}, & \left.R(\lambda, T)\right|_{X_{1}}=\left.0\right|_{X_{1}}, \\
R(\lambda, T)\left(X_{2}\right) \subset X_{2}, & \left.R(\lambda, T)\right|_{X_{2}}=\left.\left(\lambda I_{2}-A_{2}\right)^{-1}\right|_{X_{2}},
\end{array}
$$

whenever $|\lambda| \geq r$. 
Set $0_{1}=\left.0\right|_{X_{1}}$. Let $u \in D(T)$ and let $v \in X$ with $J_{0} v=T u$. Then we have $R(\lambda, T)(\lambda u-v)=u$ for a fixed $\lambda$ with $|\lambda| \geq r$. Write $u=u_{1}+u_{2}, v=v_{1}+v_{2}$, with $u_{j}, v_{j} \in X_{j}$ for $j=1,2$. Using (3), we have in fact that

$$
R(\lambda, T)\left(\lambda u_{1}-v_{1}\right)=0=u_{1} \quad \text { and } \quad R(\lambda, T)\left(\lambda u_{2}-v_{2}\right)=u_{2} .
$$

These relations imply that

$$
\begin{aligned}
v_{1} \in N\left(0_{1}\right) & =X_{1}, \\
\left(\lambda I_{2}-A_{2}\right)^{-1}\left(\lambda u_{2}-v_{2}\right) & =u_{2},
\end{aligned}
$$

From (5) we obtain that $A_{2} u_{2}=v_{2}$. This calculation shows that $D(T) \subset\{0\} \oplus X_{2}$, and that $T\left(0 \oplus u_{2}\right)=v_{1}+A_{2} u_{2}+X_{0}$ whenever $0 \oplus u_{2} \in D(T)$.

If $u=0$, then we may take as $v \in X$ with $J_{0} v=T u=0$ any vector $v \in X_{0}$. The decomposition $0=u_{1}+u_{2}$ shows that $u_{1}=u_{2}=0$. Then, from (4) and (5) we derive $v_{1} \in X_{1}$ and $v_{2}=0$. Therefore, $X_{0} \subset X_{1}$. As $T\left(0_{1} v_{1} \oplus 0\right)=0=v_{1}+X_{0}$ for every $v_{1} \in X_{1}$, we must have $X_{0}=X_{1}$.

In fact, $D(T)=\{0\} \oplus X_{2}$. Indeed, if $A_{2} u_{2}=v_{2}$ for some $u_{2} \in X_{2}$, taking into account (5), we have

$$
\left(\lambda I_{2}-A_{2}\right)^{-1}\left(\lambda u_{2}-v_{2}\right)=u_{2}=R(\lambda, T)\left(\lambda u_{2}-v_{2}\right) \in D(T) .
$$

In summary, we have now two closed vector subspaces $X_{1}$ and $X_{2}$ of $X$ with $X=X_{1} \oplus X_{2}$, the operator $0_{1} \in \mathscr{B}\left(X_{1}\right)$, an operator $A_{2} \in \mathscr{B}\left(X_{2}\right), \quad X_{0}=X_{1}$, $D(T)=\{0\} \oplus X_{2}$, and $T:\{0\} \oplus X_{2} \mapsto\left(X_{1} \oplus X_{2}\right) / X_{1}$ is given by $T\left(0 \oplus x_{2}\right)=$ $0 \oplus A_{2} x_{2}+X_{1}$ for all $0 \oplus x_{2} \in\{0\} \oplus X_{2}$. Setting $T_{1}:\{0\} \subset X_{1} \mapsto X_{1} / X_{1}=\{0\}$ and $T_{2}=A_{2}: X_{2} \mapsto X_{2}$, we obtain $T=T_{1} \oplus_{q} T_{2}$. Assuming $X_{1} \neq\{0\}$, we must have $\sigma(T) \ni\{\infty\}$ via Definition 2, which is not possible. Therefore, which is not possible. Therefore, $X_{1}=\{0\}$, and so $T=A_{2} \in \mathscr{B}\left(X_{2}\right)=\mathscr{B}(X)$.

Conversely, the conditions in the statement from above are obviously sufficient to insure the boundedness of the spectrum of $T$.

Remark 12. From the previous proof it follows that if $\infty$ is 0 -regular for $T$, then $T=T_{1} \oplus_{q} T_{2}$, where $T_{1}:\{0\} \subset X_{1} \mapsto+X_{1} / X_{1}=\{0\}$, and $T_{2}: X_{2} \mapsto X_{2}$ is bounded.

Corollary 13. Let $T: D(T) \subset X \mapsto X / X_{0}$ be closed. Then $\sigma(T)=\sigma_{A}(T)$ if and only if $T \in \mathscr{B}(X)$, and $\sigma(T)=\sigma_{A}(T) \cup\{\infty\}$ otherwise.

In particular, if $T: D(T) \subset X \mapsto X$ is a closed operator, the spectrum of $T$ is a bounded subset of $\mathbb{C}$ if and only if $T \in \mathscr{B}(X)$.

The next result is related to [Baskakov and Chernyshov 2002, Lemma 2.2].

Corollary 14. Let $Z \subset X \times X$ be a closed relation. The spectrum of $Z$ is a bounded subset of $\mathbb{C}$ if and only if $Z$ is the graph of an operator in $\mathscr{B}(X)$. 
The spectrum of a direct sum of two quotient range operators behaves as one expects (see also Lemma 2.1 from [Baskakov and Chernyshov 2002], in the context of linear relations):

Corollary 15. If $T: D(T) \subset X \mapsto X / X_{0}$ is closed and has the form $T=T_{1} \oplus_{q} T_{2}$, then $\sigma(T)=\sigma\left(T_{1}\right) \cup \sigma\left(T_{2}\right)$.

Proof. Note that $J_{0}=J_{01} \oplus_{q} J_{02}$, where $J_{0 j}: X_{j} \mapsto X_{j} / X_{0 j}$ are the canonical projections for $j=1,2$. We have to show that $\rho(T)=\rho\left(T_{1}\right) \cap \rho\left(T_{2}\right)$. We have the following cases.

First, fix $\lambda \in \rho(T) \cap \mathbb{C}$. Setting $S=\lambda J_{0}-T, S_{j}=\lambda J_{0 j}-T_{j}, j=1,2$, we have to show that $S=S_{1} \oplus_{q} S_{2}$ is bijective if and only if both $S_{1}, S_{2}$ are bijective, which is routine and is left to the reader. In fact, we obtain that

$$
\left(\lambda J_{0}-T\right)^{-1}=\left(\left(\lambda J_{01}-T_{1}\right)^{-1} \oplus\left(\lambda J_{02}-T_{2}\right)^{-1}\right) V^{-1},
$$

where $V$ is given by (1). Therefore,

$$
R(\lambda, T)=R\left(\lambda, T_{1}\right) \oplus R\left(\lambda, T_{2}\right) .
$$

This clearly shows that $\sigma_{A}(T)=\sigma_{A}\left(T_{1}\right) \cup \sigma_{A}\left(T_{2}\right)$.

Second, we have only to note that $T \in \mathscr{B}(X)$ if and only if $T_{j} \in \mathscr{B}\left(X_{j}\right)(j=1,2)$, which easily leads to the equality $\sigma(T)=\sigma\left(T_{1}\right) \cup \sigma\left(T_{2}\right)$, via Corollary 13 .

A general result concerning the existence of an analytic functional calculus for quotient range closed operators is the following.

Theorem 16. For every quotient range closed operator $T$ with $\varnothing \neq \rho(T)$, the map $f \mapsto f(T)$ of $\mathcal{O}(T)$ into $\mathscr{B}(X)$ is a unital algebra morphism. If $\sigma(T)$ is bounded, then $T \in \mathscr{B}(X)$ and $p_{1}(T)=T$, where $p_{1}(\lambda)=\lambda$ for all $\lambda \in \mathbb{C}$.

Proof. If $\sigma(T)$ is unbounded, the assertion follows from Proposition 10. If $\sigma(T)$ is bounded, then $T \in \mathscr{B}(X)$ by Proposition 11, and the assertion is classical.

Remark 17. (i) For every $f \in \mathcal{O}(T)$, we have

$$
\left.f(T)\right|_{X_{0}}= \begin{cases}\left.f(T)\right|_{\{0\}}=0 & \text { if } \infty \notin \sigma(T), \\ f(\infty) I & \text { if } \infty \in \sigma(T) .\end{cases}
$$

Indeed, we clearly have

$$
\int_{\Gamma} f(\lambda) R(\lambda, T) d \lambda=\left(\int_{\Gamma} f(\lambda)\left(\lambda J_{0}-T\right)^{-1} d \lambda\right) J_{0},
$$

for each admissible contour $\Gamma$ surrounding $\sigma(T)$, which in turn implies the desired equalities. 
This remark also shows that $f(T)\left(X_{0}\right) \subset X_{0}$ for every $f \in \mathcal{O}(T)$. This allows us to define an operator $f^{\circ}(T) \in \mathscr{B}\left(X / X_{0}\right)$, given by

$$
f^{\circ}(T)\left(x+X_{0}\right):=f(T) x+X_{0} \text { for } x \in X,
$$

for all $f \in \mathcal{O}(T)$. In other words, $f^{\circ}(T) J_{0}=J_{0} f(T)$ for all $f \in \mathscr{O}(T)$.

(ii) If $\infty$ is an isolated point of $\sigma(T)$, then $E=(2 \pi i)^{-1} \int_{\Gamma} f(\lambda) R(\lambda, T) d \lambda$ is a projection, where $\Gamma$ is a contour surrounding $\sigma_{A}(T)$.

(iii) If $Z \subset X \times X$ is a closed relation with nonempty resolvent set, we may define the operator $f(Z):=f\left(Q_{Z}\right)$ for every analytic function from $\mathcal{O}(Z):=\mathcal{O}\left(Q_{Z}\right)$ (see Remark 4(ii)). This provides an analytic functional calculus for $Z$, whose properties are easily derived from those valid for $Q_{Z}$ (see also [Baskakov and Chernyshov 2002, formula (2.8)] for a similar but partial approach.)

\section{Quotient range operators with unbounded spectrum}

As before, let $X$ be a complex Banach space, let $X_{0}$ be a closed vector subspace of $X$, and let $J_{0}: X \mapsto X / X_{0}$ be the canonical projection. Let also $T: D(T) \subset$ $X \mapsto X / X_{0}$ be closed. We may consider on $D(T)$ the graph norm given by

$$
\|x\|_{T}:=\|x\|+\inf _{J_{0} y=T x}\|y\| \text { for } x \in D(T) .
$$

It is well known that when endowed with this norm, the vector space $D(T)$ becomes a Banach space; see for instance [Cross 1998, Section IV.3]. With the terminology from [Waelbroeck 1982], $\left(D(T),\|\cdot\|_{T}\right)$ becomes a Banach subspace of $X$, which will be occasionally denoted by $D_{T}$.

It is obvious that the maps $T: D_{T} \mapsto X / X_{0}$ and $J_{T}: D_{T} \mapsto X / X_{0}$, with $J_{T}=\left.J_{0}\right|_{D_{T}}$, are continuous.

Throughout this section, $T: D(T) \subset X \mapsto X / X_{0}$ will be a closed (quotient range) operator, with $\infty \in \sigma(T)$ and a nonempty resolvent set.

Lemma 18. For every function $f \in \mathcal{O}(T)$ and each admissible contour $\Gamma$ surrounding $\sigma(T)$, the map

$$
X \ni x \mapsto \int_{\Gamma} f(\lambda) R(\lambda, T) x d \lambda
$$

has values into the Banach space $D_{T}$ and is continuous.

In particular, if $f(\infty)=0$, then $f(T)$ is a continuous operator from $X$ into $D_{T}$. Proof. Indeed, $R(\lambda, T)=\left(\lambda J_{0}-T\right)^{-1} J_{0}: X \mapsto D_{T}$ is in $\mathscr{B}\left(X, D_{T}\right)$ for all $\lambda \in \rho(T)$, and hence

$$
2 \pi i(f(T)-f(\infty))=\int_{\Gamma} f(\lambda)\left(\lambda J_{0}-T\right)^{-1} J_{0} d \lambda \in \mathscr{B}\left(X, D_{T}\right),
$$


which implies the assertions.

We recall that for any quotient range operator $T$ and each function $f \in \mathcal{O}(T)$, we denote by $f^{\circ}(T)$ the operator induced by $f(T)$ in $X / X_{0}$; see Remark 17(i).

Lemma 19. Let $f \in \mathcal{O}(T)$ be such that $f_{1}(\lambda)=\lambda f(\lambda) \in \mathcal{O}(T)$. Then $T f(T)=$ $J_{0} f_{1}(T)=f_{1}^{\circ}(T) J_{0}$.

Proof. It is clear that $f(\infty)=0$. Let $\Gamma$ be an admissible contour surrounding $\sigma(T)$ in the domain of definition of $f$. We have

$$
\begin{aligned}
T(f(T) x) & =\frac{1}{2 \pi i} \int_{\Gamma} f(\lambda) T\left(\lambda J_{0}-T\right)^{-1} J_{0} x d \lambda \\
& =J_{0}\left(-\frac{1}{2 \pi i} \int_{\Gamma} f(\lambda) x d \lambda+\frac{1}{2 \pi i} \int_{\Gamma} \lambda f(\lambda)\left(\lambda J_{0}-T\right)^{-1} J_{0} x d \lambda\right) \\
& =J_{0}\left(f_{1}(T) x\right)=f_{1}^{\circ}(T) J_{0} x,
\end{aligned}
$$

because $-(1 / 2 \pi i) \int_{\Gamma} f(\lambda) d \lambda=f_{1}(\infty)$.

Remark 20. With the notation from the previous lemma, if $x \in D(T)$ and $y \in X$ satisfy $J_{0} y=T x$, then $f_{1}(T) x=f(T) y$. Indeed,

$$
\begin{aligned}
f_{1}(T) x & =f_{1}(\infty) x+\frac{1}{2 \pi i} \int_{\Gamma} \lambda f(\lambda)\left(\lambda J_{0}-T\right)^{-1} J_{0} x d \lambda \\
& =\frac{1}{2 \pi i} \int_{\Gamma} f(\lambda)\left(\lambda J_{0}-T\right)^{-1} J_{0} y d \lambda=f(T) y,
\end{aligned}
$$

because $(1 / 2 \pi i) \int_{\Gamma} f(\lambda) x d \lambda=-f_{1}(\infty) x$, as noticed before.

Lemma 21. For all $f \in \mathcal{O}(T)$ and $x \in D(T)$, we have $T f(T) x=f^{\circ}(T) T x$.

Proof. Because the function $\lambda f(\lambda)$ is not necessarily in $\mathcal{O}(T)$, we need an argument different from that in the proof of Lemma 19.

If $(x, y) \in G_{0}(T)$, then $J_{0} y=T x$. Therefore, for a fixed $\lambda \in \rho(T)$,

$$
T R(\lambda, T) x=-J_{0} x+\lambda J_{0}\left(\lambda J_{0}-T\right)^{-1} J_{0} x=J_{0} R(\lambda, T) y .
$$

Let $\Gamma$ be an admissible contour surrounding $\sigma(T)$ in the domain of $f \in \mathcal{O}(T)$, positively oriented. We have, via (8), that

$$
T \int_{\Gamma} f(\lambda) R(\lambda, T) x d \lambda=J_{0} \int_{\Gamma} f(\lambda) R(\lambda, T) y d \lambda,
$$

implying $T f(T) x=J_{0} f(T) y$. Consequently,

$$
T f(T) x=J_{0} f(T) y=f^{\circ}(T) J_{0} y=f^{\circ}(T) T x \quad \text { for all } x \in D(T) .
$$


The next result is a version of the idempotent theorem in the context of quotient range operators. For a similar result in the context of linear relations, see [Baskakov and Chernyshov 2002, Theorem 2.3]. Unlike the result there, our proof uses essentially Theorem 16 .

Theorem 22. Let $T: D(T) \subset X \mapsto X / X_{0}$ be a quotient range operator with $\sigma(T) \ni \infty$ and assume that there are two nonempty disjoint closed sets $F, H \subset \mathbb{C}_{\infty}$ such that $\sigma(T)=F \cup H$. Then there exist closed vector subspaces $X_{F}$ and $X_{H}$ with $X=X_{F} \oplus X_{H}$, and operators $T_{F}: D\left(T_{F}\right) \subset X_{F} \mapsto X_{F} / X_{0 F}$ and $T_{H}: D\left(T_{H}\right) \subset$ $X_{H} \mapsto X_{H} / X_{0 H}$, where $X_{0 F} \subset X_{F}, X_{0 H} \subset X_{H}$ and $X_{0}=X_{0 F} \oplus X_{0 H}$, such that $D(T)=D\left(T_{F}\right) \oplus D\left(T_{H}\right)$ and $T=T_{F} \oplus_{q} T_{H}$.

In addition, $\sigma\left(T_{F}\right)=F$ and $\sigma\left(T_{H}\right)=H$.

Proof. To fix the ideas, assume that $\infty \in F$. We choose open sets $U$ and $V$ in $\mathbb{C}_{\infty}$ such that $U \supset F, V \supset H$ and $U \cap V=\varnothing$. Then the characteristic functions $\chi_{U}$ and $\chi_{V}$ of the sets $U$ and $V$ respectively, restricted to $U \cup V$, are analytic. We put $P_{F}=\chi_{U}(T)$ and $P_{H}=\chi_{V}(T)$. Since $\chi_{U}^{2}=\chi_{U}$, and by a similar relation for $\chi_{V}$, the operators $P_{F}$ and $P_{H}$ are projections via Proposition 10. Moreover, $P_{F} P_{H}=P_{H} P_{F}=0$ and $P_{F}+P_{H}=I$.

In fact, since $\infty \in F$, we have

$$
P_{F}=I+\frac{1}{2 \pi i} \int_{\Gamma_{F}} R(\lambda, T) d \lambda, \quad \text { and } \quad P_{H}=\frac{1}{2 \pi i} \int_{\Gamma_{H}} R(\lambda, T) d \lambda,
$$

where $\Gamma_{F}$ and $\Gamma_{H}$ are admissible contours surrounding $F$ and $H$ in $U$ and $V$, respectively.

Note that $\left.P_{H}\right|_{X_{0}}=0$ and $\left.P_{F}\right|_{X_{0}}$ is the identity on $X_{0}$; see Remark 17(i).

Lemma 21 shows that if $x \in D(T)$, then $P_{F} x \in D(T)$, and $T P_{F} x=P_{F}^{\circ} T x$, where $P_{F}^{\circ}=\chi_{U}^{\circ}(T)$. Similarly, $P_{H} x \in D(T)$ and $T P_{H} x=P_{H}^{\circ} T x$. This also shows that $D(T)=\left(D(T) \cap P_{F}(X)\right) \oplus\left(D(T) \cap P_{F}(H)\right)$.

Let $X_{F}=P_{F}(X)$ and $X_{H}=P_{H}(X)$. Obviously, $X=X_{F} \oplus X_{H}$. We have $X_{0} \subset X_{F}$, and we put $X_{0 F}=X_{0}$ and $X_{0 H}=\{0\}$.

Let $T_{F}=\left.T\right|_{\left(D(T) \cap X_{F}\right)}$. For each $x \in D\left(T_{F}\right):=D(T) \cap X_{F}$, Lemma 21 gives $T_{F} x \in X_{F} / X_{0 F}$. Similarly, if $T_{H}=\left.T\right|_{\left(D(T) \cap X_{H}\right)}$ for each $x \in D\left(T_{H}\right):=D(T) \cap X_{H}$, we have $T_{H} x \in X_{H} / X_{0 H}=X_{H}$. Consequently,

$$
T\left(x_{F} \oplus x_{H}\right)=T_{F}\left(x_{F}\right) \oplus T_{H}\left(x_{H}\right) \in\left(X_{F} /\left(X_{0 F}\right) \oplus\left(X_{H} /\left(X_{0 H}\right)\right.\right.
$$

for all $x_{F} \in D\left(T_{F}\right)$ and $x_{H} \in D\left(T_{H}\right)$, and so $T=T_{F} \oplus_{q} T_{H}$.

Let us show that $\sigma\left(T_{F}\right) \subset F$.

Let $\mu \in \mathbb{C} \backslash F$. With no loss of generality we may suppose that $\mu \notin U$. Then the function $f_{\mu}(\lambda)=(\mu-\lambda)^{-1} \chi_{U}(\lambda)$ is analytic in $U \cup V$, null at infinity, and we 
may define the operator

$$
f_{\mu}(T)=\frac{1}{2 \pi i} \int_{\Gamma_{F}} f_{\mu}(\lambda) R(\lambda, T) d \lambda=P_{F} f_{\mu}(T) .
$$

Because we have $(\mu-\lambda) f_{\mu}(\lambda)=\chi_{U}(\lambda)$, it follows that $\mu f_{\mu}(T)-f_{1, \mu}(T)=P_{F}$, where $f_{1, \mu}(\lambda)=\lambda f_{\mu}(\lambda) \in \mathcal{O}(T)$.

Let us show that $\mu J_{F}-T_{F}$ is injective, where $J_{F}: X_{F} \mapsto X_{F} / X_{0}$ is $\left.J_{0}\right|_{X_{F}}$. Assuming that for an $x \in D\left(T_{F}\right)$ one has $\mu J_{F} x=T_{F} x$, and fixing an $y \in X_{F}$ with $J_{F} y=T_{F} x$, we have $\mu x-y \in X_{0}$. Because $f_{\mu}(\infty)=0$, we infer that

$$
\begin{aligned}
0 & =f_{\mu}(T)(\mu x-y)=\frac{1}{2 \pi i} \int_{\Gamma_{F}} f_{\mu}(\lambda)\left(\lambda J_{0}-T\right)^{-1} J_{0}(\mu x-y) d \lambda \\
& =\frac{1}{2 \pi i} \int_{\Gamma_{F}}\left(\left(\chi_{U}(\lambda) R(\lambda, T) x+f_{1, \mu}(\lambda) R(\lambda, T) x-f_{\mu}(\lambda)\left(\lambda J_{0}-T\right)^{-1} J_{0} y\right) d \lambda\right. \\
& =P_{F} x=x,
\end{aligned}
$$

where we have used the equality $f_{1, \mu}(T) x=f_{\mu}(T) y$, via Remark 28 .

Let us show that $\mu J_{F}-T_{F}$ is surjective. Let $y=P_{F} y \in X_{F}$. Note that $y=$ $\mu f_{\mu}(T) y-f_{1, \mu}(T) y$, as we have seen above. Moreover, by Lemma $19 J_{0} y=$ $\mu J_{0} f_{\mu}(T) y-T f_{\mu}(T) y$. Therefore, $\left(\mu J_{F}-T_{F}\right)^{-1}$ exists for all $\mu \notin U$. Since $U$ is an arbitrary open neighborhood of $F$, it follows that $\left(\mu J_{F}-T_{F}\right)^{-1} J_{F}=\left.f_{\mu}(T)\right|_{X_{F}}$ for all $\mu \notin F$.

We show now that $\sigma\left(T_{H}\right) \subset H$. First of all, we identify the space $\left(X_{H}+X_{0}\right) / X_{0}$ with $X_{H}$, and so $\left.J_{0}\right|_{X_{H}}=I_{H}$, where $I_{H}$ is the identity on $X_{H}$. Note also that $T_{H}: D\left(T_{H}\right) \mapsto X_{H}$ is a simply closed operator.

Fixing $\mu \in \mathbb{C} \backslash H$, we may suppose that $\mu \notin V$. Then the function $g_{\mu}(\lambda)=$ $(\mu-\lambda)^{-1} \chi_{V}(\lambda)$ is analytic in $U \cup V$, null at infinity, and we can consider the operator $g_{\mu}(T)=P_{H} g_{\mu}(T)$.

Because we have $(\mu-\lambda) g_{\mu}(\lambda)=\chi_{V}(\lambda)$, it follows that $\mu g_{\mu}(T)-g_{1, \mu}(T)=P_{H}$, where $g_{1, \mu}(\lambda)=\lambda g_{\mu}(\lambda) \in \mathcal{O}(T)$.

Proceeding as in the previous case, we derive that $\mu I_{H}-T_{H}: D\left(T_{H}\right) \mapsto X_{H}$ is bijective. In fact, $\left(\mu I_{H}-T_{H}\right)^{-1}=\left.g_{\mu}(T)\right|_{X_{H}}$ for all $\mu \notin H$. We omit the details.

We have only to note that

$$
\left\|g_{\mu}(T)\left|X_{H}\left\|\leq \frac{1}{2 \pi \operatorname{dist}\left(\mu, \Gamma_{H}\right)} \int_{\Gamma_{H}}\right\| R(\lambda, T) \|\right| d \lambda \mid,\right.
$$

implying that $\infty$ is 0 -regular for $T_{H}$. In other words, $\sigma\left(T_{H}\right) \subset H$.

Since we already have $\sigma\left(T_{F}\right) \subset F$ and $\sigma\left(T_{H}\right) \subset H$, it suffices to prove that $\sigma\left(T_{F}\right) \cup \sigma\left(T_{H}\right)=\sigma(T)$. Indeed, this follows from Corollary 15, showing that we must have $\sigma\left(T_{F}\right)=F$ and $\sigma\left(T_{H}\right)=H$. 
A result similar to [Baskakov and Chernyshov 2002, Theorem 2.3] follows directly from the previous theorem:

Corollary 23. Let $Z \subset X \times X$ be a closed relation with $\sigma(Z) \ni \infty$. Assume that there are two nonempty disjoint closed sets $F, H \subset \mathbb{C}_{\infty}$ such that $\sigma(Z)=F \cup H$. Then we have a decomposition $Z=Z_{F} \oplus Z_{H}$ with $Z_{F}$ and $Z_{H}$ closed relations and $\sigma\left(Z_{F}\right)=F$ and $\sigma\left(Z_{H}\right)=H$.

We end this section with a version of the spectral mapping theorem. A similar result valid for linear relations can be found in [Baskakov and Chernyshov 2002, Theorem 2.5], whose proof uses Gelfand's theory (see also Corollary 10 there). Our proof is different and is based on Theorems 16 and 22.

Theorem 24. For every $f \in \mathcal{O}(T)$, we have $\sigma(f(T))=f(\sigma(T))$.

Proof. Fix an $f \in \mathcal{O}(T)$. Let $\mu \notin f(\sigma(T))$ with $\mu \neq \infty$. Then the function $g_{\mu}(\lambda)=(\mu-f(\lambda))^{-1}$ is in $\mathrm{O}(T)$. It is plain that $(\mu I-f(T)) g_{\mu}(T)=I$, showing that $g_{\mu}(T)=(\mu I-f(T))^{-1}$, and so $\sigma(f(T)) \subset f(\sigma(T))$ (that it is 0-regular for $f(T)$ is obvious).

Conversely, let $\mu_{0} \in f(\sigma(T))$, so $\mu_{0}=f\left(\lambda_{0}\right)$ for some $\lambda_{0} \in \sigma(T)$. Assume that $\mu_{0} \notin \sigma(f(T))$.

In the case $\lambda_{0} \neq \infty$, we consider the function $h(\lambda)=\left(\lambda_{0}-\lambda\right)^{-1}\left(\mu_{0}-f(\lambda)\right)$, which can be clearly extended at $\lambda=\lambda_{0}$, and this extension belongs to $\mathcal{O}(T)$. Note that $\lambda_{0} h(T)-h_{1}(T)=\mu_{0} I-f(T)$, where $h_{1}(\lambda)=\lambda h(\lambda) \in \mathbb{O}(T)$. Therefore,

$$
\lambda_{0} h(T)\left(\mu_{0} I-f(T)\right)^{-1}-h_{1}(T)\left(\mu_{0} I-f(T)\right)^{-1}=I .
$$

This shows that for each $v \in X$ we have

$$
\left(\lambda_{0} J_{0}-T\right) h(T)\left(\mu_{0} I-f(T)\right)^{-1} v=J_{0} v
$$

via Lemma 19. Therefore, $\lambda_{0} J_{0}-T$ is surjective.

Further, let $x \in X$ be such that $\left(\lambda_{0} J_{0}-T\right) x=0$, and let $y \in X$ with $J_{0} y=T x$. Using (9), we have

$$
x=\left(\mu_{0} I-f(T)\right)^{-1}\left(\lambda_{0} h(T)-h_{1}(T) x\right)=\left(\mu_{0} I-f(T)\right)^{-1} h(T)\left(\lambda_{0} x-y\right)=0
$$

via Remark 20, and that $J_{0}\left(\lambda_{0} x-y\right)=0$ and $h(\infty)=0$; see also Remark 17(i). This shows that $\lambda_{0} J_{0}-T$ is injective too. Consequently, $\lambda_{0} J_{0}-T$ is invertible, which is not possible.

In the case that $\lambda_{0}=\infty$, and there exists a sequence $\left(\lambda_{m}\right)_{m \geq 1}$ in $\sigma_{A}(T)$ such that $\lim _{m \rightarrow \infty} \lambda_{m}=\lambda_{0}$, then $f\left(\lambda_{m}\right) \in \sigma(f(T))$ for all $m \geq 1$ by the first part of the proof, implying $f(\infty) \in \sigma(f(T))$.

Finally, if $\infty$ is an isolated point of $\sigma(T)$, then, according to Theorem 22, there is a decomposition $X=X_{1} \oplus X_{\infty}$, and setting $T_{\infty}=\left.T\right|_{D(T) \cap X_{\infty}}$, we have $\sigma\left(T_{\infty}\right)=$ $\{\infty\}$. Because we have $\sigma\left(f\left(T_{\infty}\right)\right) \subset f\left(\sigma\left(T_{\infty}\right)\right)=\{f(\infty)\}$ by the first part of the 
proof, we must actually have $\sigma\left(f\left(T_{\infty}\right)\right)=\{f(\infty)\}$ since $\sigma\left(f\left(T_{\infty}\right)\right)$ is nonempty. Consequently, $f(\infty) \in \sigma(f(T))$, as a consequence of Corollary 15 and of the equality $f(T)=f\left(T_{1}\right) \oplus_{q} f\left(T_{\infty}\right)$, where $T_{1}=\left.T\right|_{X_{1}} \in \mathscr{B}\left(X_{1}\right)$.

Theorem 2.5 from [Baskakov and Chernyshov 2002] is then a consequence of the preceding theorem:

Corollary 25. If $Z$ is a closed relation with nonempty resolvent set and unbounded spectrum, we have $\sigma(f(Z))=f(\sigma(Z))$ for all $f \in \mathbb{O}(Z)$.

Using Theorem 24, we get the superposition of the analytic functional calculus:

Proposition 26. Let $f \in \mathcal{O}(T)$ and let $g \in \mathbb{O}(f(T))$. Then we have $g \circ f \in \mathbb{O}(T)$ and $(g \circ f)(T)=g(f(T))$.

Proof. The property $g \circ f \in \mathbb{O}(T)$ follows easily from Theorem 24. The proof of the equality $(g \circ f)(T)=g(f(T))$ follows the lines of the similar assertion in [Vasilescu 1982, Theorem III.3.10(4)]. Specifically, we may choose an admissible contour $\Gamma$ surrounding $\sigma(T)$ such that $\Gamma_{1}=f(\Gamma)$ surrounds $\sigma(f(T))$. Then

$$
\begin{aligned}
g(f(T)) & =\frac{1}{2 \pi i} \int_{\Gamma_{1}} g(\mu) R(\mu, f(T)) d \mu \\
& =\frac{1}{2 \pi i} \int_{\Gamma_{1}} g(\mu)\left((\mu-f(\infty))^{-1} I+\frac{1}{2 \pi i} \int_{\Gamma}\left(\mu-f(\lambda)^{-1}\right) R(\lambda, T) d \lambda\right) d \mu \\
& =g(f(\infty)) I+\frac{1}{2 \pi i} \int_{\Gamma}\left(\frac{1}{2 \pi i} \int_{\Gamma_{1}} g(\mu)\left(\mu-f(\lambda)^{-1}\right) d \mu\right) R(\lambda, T) d \lambda \\
& =g(f(\infty)) I+\frac{1}{2 \pi i} \int_{\Gamma} g(f(\lambda)) R(\lambda, T) d \lambda=(g \circ f)(T),
\end{aligned}
$$

which proves the result.

A result similar to [Baskakov and Chernyshov 2002, Corollary 2.4] can be also obtained with our techniques:

Proposition 27. We have $\sigma(T)=\{\infty\}$ if and only if there is a quasinilpotent operator $Q \in \mathscr{B}(X)$ such that $T: R(Q) \mapsto X / N(Q), T(Q x)=x+N(Q)$ for $x \in X$.

Proof. Assume $\sigma(T)=\{\infty\}$. If $h(\lambda)=\lambda^{-1}(\lambda \neq 0)$, we have $h \in \mathcal{O}(T)$ and $h(\infty)=0$. Therefore, by Lemma $19, h(T) x \in D(T)$ for all $x \in X$, and $T h(T) x=$ $J_{0} h_{1}(T) x=J_{0} x$, where $h_{1}(\lambda)=1$ for all $\lambda$. Hence $h(T)=T^{-1} J_{0}$, showing that $D(T)=R(h(T))$ and $N(h(T))=X_{0}$. We have only to remark that $\sigma(h(T))=$ $h(\{\infty\})=\{0\}$, showing that $Q=h(T)$ is quasinilpotent.

Conversely, if there is a quasinilpotent operator $Q \in \mathscr{B}(X)$ such that $T: R(Q) \mapsto$ $X / N(Q), T(Q x)=x+N(Q)$ for $x \in X$, then one has $\left(\lambda J_{0}-T\right)^{-1}(y+N(Q))=$ $(\lambda Q-I)^{-1} Q y$ for all $y \in X$ and $\lambda \in \mathbb{C}$. Hence, $\sigma(T)=\{\infty\}$.

Note also that $R(\lambda, T)=(\lambda Q-I)^{-1} Q, \lambda \in \mathbb{C}$. 
Remark 28. The spectrum of the relation $Z \subset X \times X$ is equal to $\{\infty\}$ if and only if $Z$ is the reverse of the graph of a quasinilpotent operator $Q \in \mathscr{B}(X)$. This can be deduced either from the previous result or directly, from the fact that $Z^{\dagger}$ is a bounded operator and the equality

$$
\left(\lambda^{-1} I-Z^{\dagger}\right)^{\dagger}=\lambda I+\lambda^{2}(\lambda I-Z)^{\dagger} \text { for } \lambda \neq 0 \text {; }
$$

see for instance [Sandovici 2006, (2.1.2)] or [Baskakov and Chernyshov 2002, Corollary 2.4].

\section{Quotient range operators with bounded Arens spectrum}

In this section we study those quotient range operators for which the point $\infty$ is isolated and $m$-regular, for some integer $m \geq 1$. We discuss the case $m=0$ in Remark 12. Similar results for linear relations can be also found in [Baskakov and Chernyshov 2002, Section 3]. We start with a version of Proposition 27.

Proposition 29. Let $T: D(T) \subset X \mapsto X / X_{0}$ be closed with $\sigma(T)=\{\infty\}$. The point $\infty$ is $m$-regular for $T$ for some integer $m \geq 1$ if and only if there exists $Q \in \mathscr{B}(X)$ such that $Q^{m+1}=0$, and $T: R(Q) \mapsto X / N(Q)$ is given by $T(Q x)=x+N(Q)$ for all $x \in X$.

Proof. The condition is sufficient by Proposition 27. Let us prove its necessity.

With the notation from Remark 8, because $\sigma(T)=\{\infty\}$ and so $R(\lambda, T)$ should be of the form $-\sum_{k=0}^{\infty} \lambda^{k} C_{k}$ for all $\lambda \in \mathbb{C}$, we must have $C_{k}=0$ for all $k \geq m$. Therefore, $R(\lambda, T)=-\sum_{k=0}^{m-1} \lambda^{k} C_{k}$. For the rest of the proof, we sketch an algebraic argument.

For any two distinct points $\lambda$ and $\mu$ in $\mathbb{C}$, the resolvent equation shows that

$$
(\mu-\lambda) \sum_{k=0}^{m-1} \sum_{p+q=k} \lambda^{p} \mu^{q} C_{p} C_{q}=-\sum_{k=0}^{m-1}\left(\lambda^{k}-\mu^{k}\right) C_{k} .
$$

Hence

$$
\sum_{p+q=k-1}(\lambda-\mu) \lambda^{p} \mu^{q} C_{p} C_{q}=\left(\lambda^{k}-\mu^{k}\right) C_{k}
$$

whenever $1 \leq k \leq m-1$, implying by recurrence $C_{0} C_{k-1}=C_{k}$, and so $C_{k}=C_{0}^{k+1}$. Therefore, taking $Q=C_{0}$, we must have $Q^{m+1}=C_{m}=0$.

Finally, since $R(\lambda, T)=Q(\lambda Q-I)^{-1}$, we infer the equality, $T^{-1} J_{0}=Q$, showing that $X_{0}=N(Q), D(T)=R(Q)$, and $T Q x=x+N(Q)$ for all $x \in X$.

The next result is related to [Baskakov and Chernyshov 2002, Theorem 3.1].

Theorem 30. Let $T: D(T) \subset X \mapsto X / X_{0}$ be closed, with $\sigma_{A}(T)$ bounded and $\infty \in \sigma(T)$. The point $\infty$ is $m$-regular for some integer $m \geq 1$ if and only if there 
are closed vector subspaces $X_{1}$ and $X_{2}$ of $X$ with $X=X_{1} \oplus X_{2}$, an operator $A_{1} \in \mathscr{B}\left(X_{1}\right)$ with $A_{1}^{m+1}=0$, another operator $A_{2} \in \mathscr{B}_{3}\left(X_{2}\right)$, with $X_{0}=N\left(A_{1}\right) \oplus\{0\}$, $D(T)=R\left(A_{1}\right) \oplus X_{2}$, and $T=T_{1} \oplus_{q} T_{2}$, where $T_{1}\left(A_{1} x_{1}\right)=x_{1}+N\left(A_{1}\right)$ for all $x_{1} \in X_{1}$, and $T_{2}=A_{2}$.

In addition, $\sigma_{A}(T)=\sigma\left(A_{2}\right)$.

Proof. Assume that $T$ is closed, with $\sigma_{A}(T)$ bounded, such that the point $\infty$ is $m$ regular for some integer $m \geq 1$. Then $\sigma(T)=F \cup\{\infty\}$, where $F:=\sigma_{A}(T)$. Since $F$ is bounded, according to Theorem 22 and Proposition 27, there exist closed vector subspaces $X_{F}$ and $X_{\infty}$ with $X=X_{F} \oplus X_{\infty}$, and operators $T_{F}: X_{F} \mapsto X_{F}$ and $T_{\infty}: D\left(T_{\infty}\right) \subset X_{\infty} \mapsto X_{\infty} / X_{0 \infty}$, with $\sigma\left(T_{F}\right)=F$ and $\sigma\left(T_{\infty}\right)=\{\infty\}$, where $X_{0 \infty}=N\left(Q_{\infty}\right)=X_{0}, D\left(T_{\infty}\right)=R\left(Q_{\infty}\right) \oplus X_{F}$, and $Q_{\infty} \in \mathscr{B}\left(X_{\infty}\right)$ is quasinilpotent. Moreover, $T_{\infty}\left(Q_{\infty} x\right)=x+N\left(Q_{\infty}\right)$ for all $x \in X_{\infty}$, and $T=T_{\infty} \oplus_{q} T_{F}$. In fact, since $\infty$ is $m$-regular for $T$, it is also $m$-regular for $T_{\infty}$. Therefore, $Q_{\infty}^{m+1}=0$ by Proposition 29. The assertion from the statement is obtained for $A_{1}=Q_{\infty}$ and $A_{2}=T_{F}$.

Conversely, if $T=T_{1} \oplus_{q} T_{2}$ with the stated properties, then $\sigma\left(T_{1}\right)=\{\infty\}$ and $\infty$ is $m$-regular for $T_{2}$ by Proposition 29, and so $\sigma_{A}(T)=\sigma\left(T_{2}\right)$ is bounded and $\infty$ is $m$-regular also for $T$, by (7).

A part of [Baskakov and Chernyshov 2002, Theorem 3.1] is now obtained as a consequence of the previous theorem.

Corollary 31. Given a closed linear relation $Z \subset X \times X$ with $\sigma_{A}(Z)$ a bounded subset of $\mathbb{C}$ and $\infty$ not 0 -regular, the set $\left\{|\lambda|^{1-m}\left\|(\lambda-Z)^{\dagger}\right\| ;|\lambda| \geq r\right\}$ is bounded for an integer $m \geq 1$ and some $r>\sup \left\{|\lambda| ; \lambda \in \sigma_{A}(Z)\right\}$ if and only if there exist closed linear subspaces $X_{1}$ and $X_{2}$ with $X_{1} \oplus X_{2}=X$, and operators $A_{1} \in \mathscr{B}_{(}\left(X_{1}\right)$ with $A_{1}^{m+1}=0$, and $A_{2} \in \mathscr{B}\left(X_{2}\right)$, such that

$$
Z=G\left(A_{1}\right)^{\dagger} \oplus G\left(A_{2}\right) .
$$

In this case, one has $\sigma_{A}(Z)=\sigma\left(A_{2}\right)$.

Example 32. Let $P \in \mathscr{B}(X)$ be a proper projection, and let $Z=G(P)^{\dagger}$. Clearly $Z^{\dagger}=P$ and thus $0 \in \rho(Z)$, and so $Z^{\dagger}$ is neither injective nor surjective. In fact, we can now easily compute the spectrum of $Z$. Setting $X_{1}=N(P)$ and $X_{2}=R(P)$, we have that $X=X_{1} \oplus X_{2}$. Therefore $Z=G\left(0_{1}\right)^{\dagger} \oplus G\left(I_{2}\right)$, where $0_{1}$ is the null operator on $X_{1}$ and $I_{2}$ is the identity on $X_{2}$. Using Corollary 31, it follows that $\sigma(Z)=\sigma(\{0\}) \cup \sigma\left(I_{2}\right)=\{\infty\} \cup\{1\}$.

Remark 33. Let $Z$ be a densely defined closed linear relation such that, for some $r>0$, we have $\{\lambda ;|\lambda|>r\} \subset \rho_{A}(Z)$ and $\mathscr{R}=\left\{(\lambda I-Z)^{\dagger} ;|\lambda|>r\right\}$ is a bounded subset of $\mathscr{B}(X)$. Then $\sigma_{A}(Z)$ is bounded, possibly empty. Let us show that $\sigma_{A}(Z)$ is nonempty. If $\infty$ is 0 -regular, the assertion follows via Corollary 14 (see also 
Remark 12). Assuming that $\sigma_{A}(Z)$ is empty and $\infty$ is not 0-regular, Corollary 31 shows that $\sigma\left(A_{2}\right)$ is empty, leading to $X_{2}=\{0\}$, and $A_{1}^{2}=0$. Since $D(Z)=R\left(A_{1}\right)$ is dense, the closure of $R\left(A_{1}\right)$ should be equal to $X$. Therefore $A_{1}=0$ implying $R\left(A_{1}\right)=\{0\}$, and so $X=\{0\}$, which is not possible. Consequently, $\sigma_{A}(Z)$ is nonempty.

One can see that the conditions from above on $Z$ are more general than those from [Cross 1998, Theorem VI.3.3], leading to the same conclusion.

\section{Applications to Arens polynomial calculus}

Given the linear relations $Z, Z_{1}, Z_{2}$ in $X \times X$, and $\alpha \in \mathbb{C}$, we may consider, as usual (see e.g., [Arens 1961; Cross 1998]), the following linear relations in $X$. The composition of $Z_{1}$ and $Z_{2}$ :

$$
Z_{1} \circ Z_{2}=\left\{(u, w) \in X \times X ;(u, v) \in Z_{2},(v, w) \in Z_{1} \text { for some } v \in X\right\},
$$

which will be also denoted by $Z_{1} Z_{2}$. The sum of $Z_{1}$ and $Z_{2}$ :

$$
Z_{1}+Z_{2}=\left\{(u, v+w) ; u \in D\left(Z_{1}\right) \cap D\left(Z_{2}\right),(u, v) \in Z_{1},(u, w) \in Z_{2}\right\} .
$$

The product of $Z$ by a number $\alpha \in \mathbb{C}$ :

$$
\alpha Z=\{(u, \alpha v) ;(u, v) \in Z\}=\alpha I \circ Z,
$$

where we identify the operator $\alpha I$ with its graph. Note that $Z_{1}+Z_{2}$ is not an algebraic sum and that $0 Z$ is the null operator on $D(Z)$.

For a linear relation $Z \subset X \times X$ we write

$$
Z^{n}:=\underbrace{Z \circ Z \circ \cdots \circ Z}_{\mathrm{n}} \text { for } n \in \mathbb{N}^{*} .
$$

If $p(z)=\alpha_{0}+\alpha_{1} z+\cdots+\alpha_{n} z^{n}$ for $z \in \mathbb{C}$, following Arens [1961] we define the relation

$$
p_{A}(Z):=\alpha_{0} I+\alpha_{1} Z+\cdots+\alpha_{n} Z^{n} .
$$

Remark 34. Let $Z, Z_{1}$ and $Z_{2}$ be linear relations defined on a linear space $X$. The following assertions, which are well known, follow by a simple calculation.

(i) For any $\xi, \eta \in \mathbb{C}$, one has that $(\xi I-Z)(\eta I-Z)=(\eta I-Z)(\xi I-Z)$.

(ii) $\left(Z_{1} Z_{2}\right)^{\dagger}=Z_{2}^{\dagger} Z_{1}^{\dagger}$.

We recall that the symbol $\sigma_{A}(Z)$ denotes the Arens spectrum of the linear relation $Z$; see Remark 4(ii). We also define $\rho_{A}(Z):=\mathbb{C} \backslash \sigma_{A}(Z)$.

The next proposition enables us to apply the results from the previous sections to linear relations of the form $p_{A}(Z)$; see also [Kascic 1968, Theorem 3.16]. 
Proposition 35. If $Z$ is a closed linear relation on the Banach space $X$ such that $\rho_{A}(Z) \neq \varnothing$ and $p$ is a polynomial, then $p_{A}(Z)$ is a closed linear relation on $X$.

Proof. Fix a $\lambda \in \rho_{A}(Z)$, so $(\lambda I-Z)^{\dagger} \in \mathscr{B}(X)$. Using [Brezis 1983, Theorem III.9], we obtain that $(\lambda I-Z)^{\dagger}$ is continuous from $\left(X, \sigma\left(X, X^{\prime}\right)\right)$ to $\left(X, \sigma\left(X, X^{\prime}\right)\right)$. Therefore we can finish by applying [Kascic 1968, Theorem 3.16].

The next results show that the functional calculus introduced in Theorem 16 agrees, in some sense, with the Arens polynomial calculus.

Remark 36. Let $Z$ be a closed linear relation in $X$ such that $\sigma(Z)=\{\infty\}$ and the point $\infty$ is $m$-regular for some integer $m \geq 1$. Let us compute $p_{A}(Z)$, where $p(z)=\alpha_{0}+\alpha_{1} z+\cdots+\alpha_{n} z^{n}$ for $z \in \mathbb{C}$. According to Corollary 31 (see also Remark 12), there exists $Q \in \mathscr{B}(X)$ such that $Q^{m+1}=0$ and $Z=G(Q)^{\dagger}$. Hence $Z^{k}=G\left(Q^{k}\right)^{\dagger}$ for all integers $k \geq 0$. In particular, $Z^{k}=G(0)^{\dagger}$ if $k \geq m+1$. In other words, $p_{A}(Z)=p_{A}\left(G(Q)^{\dagger}\right)$. Therefore, if $n=0$ we have $p_{A}(Z)=\alpha_{0} G(I)$; if $1 \leq n \leq m$ we have

$$
\begin{aligned}
p_{A}(Z) & =\alpha_{0} G(I)+\alpha_{1} G(Q)^{\dagger}+\cdots+\alpha_{n} G\left(Q^{n}\right)^{\dagger} \\
& =\left\{\left(x_{0}, \alpha_{0} x_{0}+\alpha_{1} x_{1}+\cdots+\alpha_{n} x_{n}\right) ; x_{0}=Q x_{1}=\cdots=Q^{n} x_{n}\right\} ;
\end{aligned}
$$

and if $n \geq m+1$,

$$
p_{A}(Z)=\left\{\left(0, \alpha_{1} x_{1}+\cdots+\alpha_{m} x_{m}+y_{m}\right) ; Q x_{1}=\cdots=Q^{n} x_{n}=0, y_{m} \in X\right\},
$$

Proposition 37. Let $Z$ be a closed linear relation in $X$ such that the point $\infty$ is $m$-regular for $Z$ for some integer $m \geq 1$. Then there exist closed linear subspaces $X_{1}$ and $X_{2}$ with $X_{1} \oplus X_{2}=X$, and operators $A_{1} \in \mathscr{B}\left(X_{1}\right)$ with $A_{1}^{m+1}=0$, and $A_{2} \in \mathscr{B}\left(X_{2}\right)$, such that

$$
p_{A}(Z)=p_{A}\left(G\left(A_{1}\right)^{\dagger}\right) \oplus G\left(p_{A}\left(A_{2}\right)\right),
$$

with $p_{A}\left(G\left(A_{1}\right)^{\dagger}\right)$ computed as in Remark 36.

Proof. If $Z=Z_{1} \oplus Z_{2}$, then $p_{A}(Z)=p_{A}\left(Z_{1}\right) \oplus p_{A}\left(Z_{2}\right)$. In particular, using $Z_{1}=G\left(A_{1}\right)^{\dagger}$ and $Z_{2}=G\left(A_{2}\right)$ obtained by Corollary 31 (see also Remark 12), we deduce the formula from the statement. Clearly, the computation of $p_{A}\left(G\left(A_{1}\right)^{\dagger}\right)$ is given by Remark 36 for $Q=A_{1}$.

Proposition 38. Let $Z$ be a closed linear relation with $\sigma(Z) \ni \infty$, and let $f \in \mathcal{O}(Z)$. Assume that $f_{n}(\lambda)=\lambda^{n} f(\lambda) \in \mathbb{O}(Z)$, where $n \geq 1$ is an integer. Then we have $(f(Z) x,(p f)(Z) x) \in p_{A}(Z)$ for all polynomials $p$ of degree $n$ and all vectors $x \in X$.

Proof. Set $f_{k}(\lambda)=\lambda^{k} f(\lambda) \in \mathcal{O}(Z)$ for $1 \leq k \leq n$. It follows, as in Lemma 19, that $\left(f(Z) x, f_{1}(Z) x\right) \in Z$. Similarly, $\left(f_{k-1}(Z) x, f_{k}(Z) x\right) \in Z$ for all $k=2, \ldots, n$. Consequently, $\left(f(Z) x, f_{k}(Z) x\right) \in Z^{k}$ for all $k=1, \ldots, n$. 
If $p(z)=a_{0}+a_{1} z+\cdots+a_{n} z^{n}(z \in \mathbb{C})$, then

$(f(Z) x,(p f)(Z) x)=\left(f(Z) x, a_{0} f(Z) x+a_{1} f_{1}(Z) x+\cdots+a_{n} f_{n}(Z) x \in p_{A}(Z)\right.$.

We have the following spectral mapping theorem for polynomials.

Proposition 39. Let $Z$ be a closed linear relation on the Banach space $X$ such that $\rho(Z) \neq \varnothing$ and let $p$ be a nonconstant polynomial.

(i) $\sigma_{A}\left(p_{A}(Z)\right)=p\left(\sigma_{A}(Z)\right)$.

(ii) If $\infty \in \sigma\left(p_{A}(Z)\right)$, then $\infty \in \sigma(Z)$. Conversely, if $\infty \in \sigma(Z)$ and $\infty$ is not isolated in $\sigma(Z)$, then $\infty \in \sigma\left(p_{A}(Z)\right)$.

Proof. (i) This part follows with minor changes as [Arens 1961, Theorem 2.5]. For this reason, we omit the details.

(ii) Assume that $\infty \in \sigma\left(p_{A}(Z)\right)$. Assuming $\infty \notin \sigma(Z)$, we deduce that $Z=G(T)$, with $T \in \mathscr{B}(X)$, via Corollary 14. In this case, as we have $p_{A}(Z)=G\left(p_{A}(T)\right)$ and $p_{A}(T) \in \mathscr{B}(X)$, we infer that $\infty \notin \sigma\left(p_{A}(Z)\right)$, which is not possible.

Conversely, assume that $\infty \in \sigma(Z)$ and that $\infty$ is not isolated in $\sigma(Z)$. Then we can find a sequence $\left(\lambda_{n}\right)_{n}$ in $\sigma(Z)$ such that $\lim _{n \rightarrow \infty} \lambda_{n}=\infty$. Since $\mu_{n}=$ $p_{A}\left(\lambda_{n}\right) \in \sigma(p(Z))$ for all $n$ by (i), it follows that $\infty=\lim _{n \rightarrow \infty} \mu_{n} \in \sigma\left(p_{A}(Z)\right)$.

Remark. If $Z=\{0\} \times X$ and $p(z)=\alpha_{0}$, then $\sigma(Z)=\{\infty\}$, while $\sigma\left(p_{A}(Z)\right)=$ $\sigma\left(\alpha_{0} I\right)=\left\{\alpha_{0}\right\}$. In other words, there is a linear relation $Z$ with $\infty$ isolated in $\sigma(Z)$ such that $\infty \notin \sigma\left(p_{A}(Z)\right)$ for some polynomial $p_{A}$.

\section{References}

[Albrecht and Vasilescu 1986] E. Albrecht and F.-H. Vasilescu, "Stability of the index of a semiFredholm complex of Banach spaces”, J. Funct. Anal. 66:2 (1986), 141-172. MR 87g:58011 Zbl 0592.47008

[Arens 1961] R. Arens, "Operational calculus of linear relations", Pacific J. Math. 11 (1961), 9-23. MR 23 \#A517 Zbl 0102.10201

[Baskakov and Chernyshov 2002] A. G. Baskakov and K. I. Chernyshov, "Spectral analysis of linear relations, and degenerate semigroups of operators", Mat. Sb. 193:11 (2002), 3-42. In Russian; translated in Sb. Math. 193:11-12 (2002), 1573-1610. MR 2004k:47001 Zbl 1085.47002

[Baskakov and Zagorskiı̌ 2007] A. G. Baskakov and A. S. Zagorskiŭ, "On the spectral theory of linear relations on real Banach spaces”, Mat. Zametki 81:1 (2007), 17-31. In Russian; translated in Math. Notes 81:1-2 (2007), 15-27. MR 2009c:47002 Zbl 1180.47005

[Brezis 1983] H. Brezis, Analyse fonctionnelle: Théorie et applications, Masson, Paris, 1983. MR 85a:46001 Zbl 0511.46001

[Cross 1998] R. Cross, Multivalued linear operators, Monographs and Textbooks in Pure and Applied Mathematics 213, Marcel Dekker, New York, 1998. MR 99j:47003 Zbl 0911.47002

[Dunford and Schwartz 1958] N. Dunford and J. T. Schwartz, Linear Operators, I: General Theory, Pure and Applied Mathematics 7, Interscience Publishers, New York, 1958. MR 22 \#8302 
[Favini and Yagi 1993] A. Favini and A. Yagi, "Multivalued linear operators and degenerate evolution equations", Ann. Mat. Pura Appl. (4) 163 (1993), 353-384. MR 94f:34116 Zbl 0786.47037

[Favini and Yagi 1999] A. Favini and A. Yagi, Degenerate differential equations in Banach spaces, Monographs and Textbooks in Pure and Applied Mathematics 215, Marcel Dekker, New York, 1999. MR 99i:34079 Zbl 0913.34001

[Gheorghe and Vasilescu 2009] D. Gheorghe and F.-H. Vasilescu, "Quotient morphisms, compositions, and Fredholm index”, Linear Algebra Appl. 431:11 (2009), 2049-2061. MR 2010i:47033 Zbl 1178.47007

[Hille and Phillips 1957] E. Hille and R. S. Phillips, Functional analysis and semi-groups, American Mathematical Society Colloquium Publications 31, American Mathematical Society, Providence, R. I., 1957. MR 19,664d Zbl 0392.46001

[Kascic 1968] M. J. Kascic, Jr., "Polynomials in linear relations", Pacific J. Math. 24 (1968), 291295. MR 36 \#5720 Zbl 0155.19004

[Sandovici 2006] A. Sandovici, Contributions to the extension theory in Hilbert spaces, thesis, University of Groningen, 2006.

[Vasilescu 1982] F.-H. Vasilescu, Analytic functional calculus and spectral decompositions, Mathematics and its Applications (East European Series) 1, D. Reidel Publishing Co., Dordrecht, 1982. MR 85b:47016 Zbl 0495.47013

[Waelbroeck 1954] L. Waelbroeck, "Le calcul symbolique dans les algèbres commutatives", $C$. $R$. Acad. Sci. Paris 238 (1954), 556-558. MR 17,512g Zbl 0055.10704

[Waelbroeck 1982] L. Waelbroeck, "Quotient Banach spaces", pp. 553-562 in Spectral theory (Warsaw, 1977), edited by W. Żelazko, Banach Center Publ. 8, PWN, Warsaw, 1982. MR 85m:46072a Zbl 0492.46012

Received March 8, 2011.

DANA GHEORghe

Military TeCHNiCAl ACADEmy

BLD - GeORGE Cosbuc, No. 81-83

RO-050141 BUCHAREST

ROMANIA

gheorghedana@yahoo.com

FLORIAN-HORIA VASILESCU

DEPARTMENT OF MATHEMATICS

UNIVERSITY OF LILLE 1

59655 VILLENEUVE D' ASCQ

FRANCE

fhvasil@math.univ-lille1.fr 


\title{
PACIFIC JOURNAL OF MATHEMATICS
}

\author{
http://pacificmath.org \\ Founded in 1951 by \\ E. F. Beckenbach (1906-1982) and F. Wolf (1904-1989)
}

\section{EDITORS}

V. S. Varadarajan (Managing Editor)

Department of Mathematics

University of California

Los Angeles, CA 90095-1555

pacific@math.ucla.edu

Vyjayanthi Chari

Department of Mathematics

University of California

Riverside, CA 92521-0135

chari@math.ucr.edu

\section{Robert Finn}

Department of Mathematics Stanford University

Stanford, CA 94305-2125

finn@math.stanford.edu

Kefeng Liu

Department of Mathematics

University of California

Los Angeles, CA 90095-1555

liu@math.ucla.edu
Darren Long

Department of Mathematics

University of California

Santa Barbara, CA 93106-3080

long@math.ucsb.edu

Jiang-Hua Lu

Department of Mathematics

The University of Hong Kong

Pokfulam Rd., Hong Kong jhlu@maths.hku.hk

Alexander Merkurjev

Department of Mathematics

University of California

Los Angeles, CA 90095-1555

merkurev@math.ucla.edu
Sorin Popa

Department of Mathematics University of California

Los Angeles, CA 90095-1555 popa@math.ucla.edu

Jie Qing

Department of Mathematics

University of California

Santa Cruz, CA 95064

qing@cats.ucsc.edu

Jonathan Rogawski

Department of Mathematics

University of California

Los Angeles, CA 90095-1555

jonr@math.ucla.edu

\section{PRODUCTION}

pacific@math.berkeley.edu

\section{SUPPORTING INSTITUTIONS}

ACADEMIA SINICA, TAIPEI

CALIFORNIA INST. OF TECHNOLOGY INST. DE MATEMÁTICA PURA E APLICADA KEIO UNIVERSITY

MATH. SCIENCES RESEARCH INSTITUTE NEW MEXICO STATE UNIV.

OREGON STATE UNIV.

\author{
STANFORD UNIVERSITY \\ UNIV. OF BRITISH COLUMBIA \\ UNIV. OF CALIFORNIA, BERKELEY \\ UNIV. OF CALIFORNIA, DAVIS \\ UNIV. OF CALIFORNIA, LOS ANGELES \\ UNIV. OF CALIFORNIA, RIVERSIDE \\ UNIV. OF CALIFORNIA, SAN DIEGO \\ UNIV. OF CALIF., SANTA BARBARA
}

\author{
UNIV. OF CALIF., SANTA CRUZ \\ UNIV. OF MONTANA \\ UNIV. OF OREGON \\ UNIV. OF SOUTHERN CALIFORNIA \\ UNIV. OF UTAH \\ UNIV. OF WASHINGTON \\ WASHINGTON STATE UNIVERSITY
}

These supporting institutions contribute to the cost of publication of this Journal, but they are not owners or publishers and have no responsibility for its contents or policies.

See inside back cover or pacificmath.org for submission instructions.

The subscription price for 2012 is US \$420/year for the electronic version, and \$485/year for print and electronic.

Subscriptions, requests for back issues from the last three years and changes of subscribers address should be sent to Pacific Journal of Mathematics, P.O. Box 4163, Berkeley, CA 94704-0163, U.S.A. Prior back issues are obtainable from Periodicals Service Company, 11 Main Street, Germantown, NY 12526-5635. The Pacific Journal of Mathematics is indexed by Mathematical Reviews, Zentralblatt MATH, PASCAL CNRS Index, Referativnyi Zhurnal, Current Mathematical Publications and the Science Citation Index.

The Pacific Journal of Mathematics (ISSN 0030-8730) at the University of California, c/o Department of Mathematics, 969 Evans Hall, Berkeley, CA 94720-3840, is published monthly except July and August. Periodical rate postage paid at Berkeley, CA 94704, and additional mailing offices. POSTMASTER: send address changes to Pacific Journal of Mathematics, P.O. Box 4163, Berkeley, CA 94704-0163.

PJM peer review and production are managed by EditFLOW ${ }^{\mathrm{TM}}$ from Mathematical Sciences Publishers.

PUBLISHED BY PACIFIC JOURNAL OF MATHEMATICS

at the University of California, Berkeley 94720-3840

A NON-PROFIT CORPORATION

Typeset in LATEX

Copyright $(02012$ by Pacific Journal of Mathematics 


\section{PACIFIC JOURNAL OF MATHEMATICS}

Volume $255 \quad$ No. $2 \quad$ February 2012

On the local Langlands correspondences of DeBacker-Reeder and

Reeder for $\operatorname{GL}(\ell, F)$, where $\ell$ is prime

MOSHE ADRIAN

$R$-groups and parameters

281

DUBRAVKA BAN and DAVID GOLDBERG

Finite-volume complex-hyperbolic surfaces, their toroidal

305

compactifications, and geometric applications

LUCA FABRIZIO Di CERBO

Character analogues of Ramanujan-type integrals involving the Riemann 317 $\Xi$-function

ATUl DiXIT

Spectral theory for linear relations via linear operators

DANA GHEORGHE and FLORIAN-HORIA VASILESCU

Homogeneous links and the Seifert matrix

PEDRO M. GonZÁlez MANCHÓN

Quantum affine algebras, canonical bases, and $q$-deformation of arithmetical functions

HENRY H. KIM and KYU-HWAN LEE

Dirichlet-Ford domains and arithmetic reflection groups

GRANT S. LAKELAND

Formal equivalence of Poisson structures around Poisson submanifolds

IOAN MĂRCUT,

A regularity theorem for graphic spacelike mean curvature flows

BENJAMIN STUART THORPE

Analogues of level- $N$ Eisenstein series 